\title{
Famille et société. Réflexions sur l'anarchisme « conservateur » de Gustav Landauer
}

\author{
Mais le mariage, la propriété, la famille restent théoriquement intacts, \\ parce qu'ils constituent, dans la pratique, le fondement sur lequel la \\ bourgeoisie a édifié sa domination, parce que ces institutions, dans leur \\ forme bourgeoise, sont les conditions qui font du bourgeois un bourgeois \\ Karl Marx, Friedrich Engels, L'idéologie allemande ${ }^{l}$ \\ La nouvelle société que nous voulons préparer, dont nous nous \\ apprêtons à poser la première pierre, ne sera pas un retour à quelques \\ anciennes formations, elle sera l'ancien sous une nouvelle figure \\ Gustav Landauer, Appel au socialisme ${ }^{2}$
}

Tout au long de sa vie, le philosophe et anarchiste Gustav Landauer (1870-1919) s'efforça de réveiller en ses contemporains l'esprit de communauté, enfoui sous des décennies d'individualisme et étouffé par la déréliction des structures communautaires traditionnelles. En recréant des structures portées par l'aspiration de chacun, Landauer cherchait à faire advenir une société plus harmonieuse, dans laquelle les liens entre les individus seraient les vecteurs de la libération de tous, plutôt que des entraves à la liberté de chacun.

Dans cette lutte, Landauer dénonce les institutions communes qui consacrent l'individualisme en liant les individus par un contrat extérieur plutôt que par une commune aspiration. Mais il cherche en même temps à défendre ces structures qui sont, quant à elles, porteuses de cet esprit de communauté. De tous ces éléments, la famille est peut-être celui qui valut à Landauer le plus d'incompréhensions et d'hostilités chez les révolutionnaires, tant parmi ses contemporains que dans les cercles actuels où la famille continue de passer pour « le foyer de toute autorité $»^{3}$. La posture de Landauer constitue un double défi pour la pensée et l'action révolutionnaires : tout d'abord, il s'agit d'admettre cette idée - notamment inspirée de Proudhon - que « le fait de révolutionner et celui de conserver ne sont pas exclusifs $»^{4}$. Sur ce point, la pensée de Landauer met en lumière les limites d'une déconstruction indiscriminée de l'existant du point de vue de la révolution. Mais le propos de Landauer heurte également lorsqu'il prend la défense de la famille et du mariage contre les

1 Marx Karl et Engels Friedrich, L'idéologie allemande (1845-1846), Auger Henri et al. (trad.), Paris, Éditions sociales, 1976, p. 172.

2 Landauer Gustav, Aufruf zum Sozialismus (1911), Wolf Siegbert (dir.), Lich/Hessen, Verlag Edition AV, 2015, GLAS 11, p. 116. Les passages de l'Appel au socialisme sont extraits de la traduction préparée par Jean-Christophe Angaut et Anatole Lucet (parution prévue en octobre 2019 aux éditions La Lenteur). Les textes de Landauer sont cités et traduits d'après la série des œuvres choisies, éditée par Verlag Edition AV. L'acronyme GLAS est suivi du numéro de volume et de la pagination dans cette collection.

3 Gross Otto, « Comment surmonter la crise de la civilisation? » (1913), Etoré-Lortholary Jeanne (trad.), in Gross Otto, Psychanalyse et révolution, Le Rider Jacques (dir.), Paris, Éditions du Sandre, 2011, p. 97.

4 Landauer Gustav, Aufruf zum Sozialismus, op. cit., GLAS 11, p. 118. 
partisans de l' "amour libre ». Dans la polémique qui l'oppose à un petit groupe de la bohème munichoise autour de 1910, les protagonistes avancent pêle-mêle des arguments touchant à la fidélité amoureuse, à la jalousie, à la sexualité, au mariage, au statut des femmes et à l'émancipation des êtres humains. Les affects se mêlent aux arguments théoriques dans une vision d'ensemble où l'on fut souvent tenté d'opposer le groupe émancipé et progressiste des joyeux bohèmes au barbon austère et conservateur, incapable de débarrasser son anarchisme des dernières scories d'autoritarisme et de paternalisme ${ }^{5}$. Pourtant ce tableau trop lisse se craquelle dès lors que l'on analyse les arguments et qu'on les étudie dans leur singularité. Plus qu'une simple querelle de mœurs, ce qui se dessine alors est un débat fondamental et structurel opposant deux conceptions de la communauté et de la liberté, deux visions concurrentes de la manière de générer ou de régénérer les liens sociaux.

\section{Le terreau de la revalorisation de la famille : la controverse avec Gross}

Parmi les grands mouvements d'émancipation du début du $\mathrm{XX}^{\mathrm{e}}$ siècle, on se souvient généralement du tour impressionnant que prennent les revendications féministes. Fédérée autour de l'accession des femmes au droit de vote, cette lutte mettait en lumière certaines inégalités structurelles entre la condition des femmes et celle de leurs congénères masculins. L'un des objectifs était de faire sortir les femmes de l'état de tutelle factuelle et légale dans lequel les plongeait leur situation sociale et politique. Pour beaucoup de suffragettes, ces revendications devaient contribuer au rééquilibrage d'une structure familiale bancale, dans laquelle l'autorité du père ou du mari ôtait toute autonomie aux femmes. Au même moment, d'autres militantes et militants de la cause féministe doutent cependant qu'une redéfinition des statuts dans l'arène sociale et politique suffise à enrayer un système dont le fonctionnement était structurellement conçu pour et par les hommes. Contre les tentatives d'adaptation, supposées permettre aux femmes d'accéder à un statut égal à celui de l'homme, ces voix cherchent à ébranler le système patriarcal dans son ensemble, en expérimentant, d'abord à petite échelle, de nouveaux rapports entre les sexes au-delà de la famille nucléaire. En France, les «milieux libres » de la Belle époque mettent en pratique la « camaraderie amoureuse $\rangle^{6}$; de l'autre côté du Rhin, c'est tout un pan de cette génération écrasée par les diktats du «monde des pères » qui fait le choix de renoncer aux liens du mariage pour retrouver la spontanéité et l'immédiateté d'un "amour libre». Il arrive en outre que cette dénonciation du système patriarcal et de ses conséquences aliénantes (dépendance des femmes visà-vis du père puis du mari, hypocrisie concernant les relations extra ou antéconjugales...) se généralise. De même que le capitalisme semble intrinsèquement lié à l'oppression des travailleurs, la famille passe quelquefois pour le lieu d'une nécessaire oppression de ses prolétaires : les femmes. C'est l'analyse que propose Engels dans sa célèbre étude sur L'origine de la famille, de la propriété privée et de l'État, dans laquelle il affirme que «dans la famille, l'homme est le bourgeois ; la femme joue le rôle du prolétariat $»^{7}$.

5 C'est notamment l'impression qui se dégage du témoignage de Jung Franz, Le chemin vers le bas : considérations d'un révolutionnaire allemand sur une grande époque (1900-1950) (1961), Gallissaires Pierre (trad.), 2éd., Marseille, Agone, 2007, p. 73 et 75 : « Je m'étais joint au groupe Die Tat, autour d'Erich Mühsam. Nous avions Gustav Landauer pour prophète [...] Mühsam lisait des poèmes et disait quelques mots, puis on chantait et dansait. Nous avions une guitare et un harmonica et nous payions la bière - plus exactement, Mühsam payait la bière. S'il m'est encore permis de le dire, c'était une ambiance merveilleuse. Landauer, qui venait régulièrement de Francfort pour contrôler nos activités, devait interdire ces soirées quelque temps plus tard».

6 Beaudet Céline, Les milieux libres : vivre en anarchiste à la Belle époque en France, Saint-Georges-d'Oléron, Les Éditions libertaires, 2006.

7 Engels Friedrich, L'origine de la famille, de la propriété privée et de l'État (1884), Stern Jeanne (trad.), Paris, Éditions sociales, 1971, p. 72. 
Une telle analyse fait de la famille nucléaire non seulement l'expression tardive d'une organisation sociale propre à la modernité, mais bien plus encore le foyer ancestral et la cause de toutes les oppressions au sein de la société bourgeoise. Dans ce référentiel elle devient - pour reprendre les termes des pères fondateurs du marxisme - « le fondement sur lequel la bourgeoisie a édifié sa domination $»^{8}$. C'est cette disqualification sans ménagement de la famille qui est au cœur de la vive polémique qui oppose, autour de 1910, Gustav Landauer à plusieurs membres de la bohème munichoise. Dans cette controverse, l'anarchiste présente tous les traits du censeur prude et réactionnaire. Pour ne rien améliorer, Landauer émaille sa défense du mariage de quelques remarques explicitement misogynes. On peut alors se demander si la liberté défendue à tous crins par le révolutionnaire ne s'arrêterait pas en fait au seuil du foyer familial, dernier bastion du conservatisme dans un monde émancipé. Pourquoi serait-il si ardu pour lui de concevoir une émancipation jusque dans les limites de la simple maison ? Les hypothèses abondent pour expliquer cette inconsistance du propos de l'anarchiste, mais toutes restent teintées d'une part plus ou moins grande de psychologisme. Il semble pourtant que la défense de la famille par Gustav Landauer prend appui sur des motifs théoriques profonds, et que ces relents de conservatisme doivent être compris autrement que comme une simple limitation de son anarchisme. Retraçons donc le cheminement qui permet de saisir, derrière les hauts cris d'une apparente affaire de mœurs, les soubassements théoriques d'une conception sociale révolutionnaire affirmant la nécessaire conservation de certaines structures.

Dans ce débat, dont l'empreinte est consignée dans les publications, lettres et journaux des différents protagonistes, Landauer fait face à un groupe solidement constitué et doté de toutes ses lettres de noblesse ès émancipation: les artistes et intellectuels du « Montmartre bavarois », le quartier de Schwabing à Munich. L'une des figures emblématiques de cette bohème est celui dont la présence retient Landauer auprès dudit groupe, le poète révolutionnaire Erich Mühsam. Ami très proche de Landauer, Mühsam s'était engagé dès 1903 en faveur de l'abrogation du paragraphe 175 du Code pénal allemand qui criminalisait l'homosexualité masculine. À Munich, il anime le groupe «Action (Tat)» de l'Alliance socialiste ${ }^{9}$. La plupart des autres groupes se trouvent en Allemagne, mais le journal Der Sozialist fait également état de membres en France, en Autriche et en Suisse. La partie helvète du projet est portée par la militante anarchiste et féministe Margarete Faas-Hardegger - la relation amoureuse qu'elle entretient pendant quelques mois avec Landauer est un élément déterminant dans la polémique dont il va être question. Il faut enfin compter sur la présence marquante de la « comtesse bohème » Franziska zu Reventlow, mère d'un enfant qu'elle éleva seule et auteure de plusieurs textes dans lesquels elle prône l'affirmation de la femme dans ce qu'elle présente comme ses deux dimensions spécifiques : la maternité et l'érotisme. Les thèses qu'elle développe à la fin du XIX ${ }^{\mathrm{e}}$ siècle dans plusieurs articles et romans se retrouvent explicitement dans les écrits des membres de cette bohème munichoise qui lui attribue le surnom de «madone païenne ».

Le quatrième membre de ce groupe hétéroclite est probablement celui dont les propos et actions provoqueront la plus vive réaction de la part de Landauer sur la question du mariage : il s'agit du sulfureux Otto Gross, psychanalyste et révolutionnaire qui fut un temps considéré par Sigmund Freud comme l'un de ses disciples les plus prometteurs aux côtés de Carl Gustav Jung. Simplement, si le père de la psychanalyse voyait dans sa discipline un moyen pour civiliser l'individu en l'accompagnant dans la répression et le refoulement de ses pulsions, Gross retint du maître sa méthode, mais pas sa finalité : dans une synthèse d'amoralisme nietzschéen, d'égoïsme stirnérien et de psychanalyse freudienne, Gross propose de faire de la cure psychanalytique un «préliminaire à

8 Marx Karl et Engels Friedrich, L'idéologie allemande, op. cit., p. 172.

9 Sur cette structure fédérative de petites communautés autonomes initiée par Landauer, voir la note 52 p. 7 [article « La controverse entre Gustav Landauer et le Vorwärts. Une querelle sur deux conceptions du socialisme. »] dans le même numéro. 
la révolution ${ }^{10}$ dont 1 'objectif serait d'œuvrer au dépassement des « effets de la collectivité sur l'individu $\gg{ }^{11}$. Il prône, au même titre que les autres protagonistes de Schwabing, une sexualité libérée des codes et des cadres imposés par la société bourgeoise. Mais Gross pousse l'argument un cran plus loin en théorisant un modèle social alternatif porté par l'affirmation suivante : «la prochaine révolution sera celle du droit matriarcal $»^{12}$.

Le psychanalyste développe sa conception dans le sillage des recherches menées par les mêmes auteurs que discute Engels dans L'origine de la famille : l'anthropologue Lewis Henry Morgan et le théoricien du matriarcat Johann Jakob Bachofen ${ }^{13}$. Comme August Bebel dans son ouvrage à succès La femme et le socialisme ${ }^{14}$, les deux chercheurs postulent l'existence d'un état antérieur - voire primitif - de la société qui serait marqué par une forme de régime matriarcal. C'est donc une idée déjà bien connue que Gross reprend à son compte, mais il fera de ce constat une injonction à recréer les conditions de ce matriarcat des origines. En outre, Gross ne voit pas dans ce schéma une simple inversion des rôles: contrairement à la gynécocratie de Bachofen, le régime matriarcal qu'il imagine propose une reconfiguration en profondeur des rapports sociaux sur une base communiste, visant à libérer les femmes de la tutelle d'un seul. Dans ce système, c'est la figure du père qui s'efface pour laisser place à une prise en charge collective des femmes, ainsi libérées des pressions individuelles que font peser sur elles un père ou un mari dont dépendrait leur propre subsistance ${ }^{15}$.

Aucune des révolutions qui appartiennent à l'histoire n'a réussi à établir la liberté de l'individualité. Elles ont toutes fait long feu, et se sont toutes achevées par une hâtive réinsertion dans la normalité généralement admise. Elles ont échoué parce que le révolutionnaire d'hier portait en lui-même l'autorité. On s'aperçoit seulement aujourd'hui que le foyer de toute autorité réside dans la famille, et que le lien entre autorité et sexualité, tel qu'il se manifeste dans la famille avec la perpétuation du droit patriarcal, asservit toute individualité. ${ }^{16}$

C'est sur cette question que se joue véritablement l'opposition entre Gustav Landauer et le groupe de Schwabing. La famille - que Gross associe à l'autorité - est conçue par l'anti-autoritaire Landauer comme le foyer et le noyau de toute liberté. Plus qu'à la simple sensibilité de chacun, c'est à leurs conceptions respectives de la liberté que tient ce désaccord : là où Gross privilégie une conception négative de la liberté en prônant une libération individuelle vis-à-vis des structures

10 Gross Otto, « Comment surmonter la crise de la civilisation? », art. cit., p. 95.

11 Gross Otto, « Les effets de la collectivité sur l'individu » (1913), Etoré-Lortholary Jeanne (trad.), in Gross Otto, Psychanalyse et révolution, Le Rider Jacques (dir.), Paris, Éditions du Sandre, 2011, pp. 99-104.

12 Gross Otto, « Comment surmonter la crise de la civilisation? », art. cit., p. 98.

13 Morgan Lewis Henry, La société archaïque (1877), Jaouiche Halie (trad.), Paris, Éditions Anthropos, 1971 ; Bachofen Johann Jakob, Le droit maternel : recherche sur la gynécocratie de l'Antiquité dans sa nature religieuse et juridique (1861), Barilier Étienne (trad.), Lausanne, L'Âge d'Homme, 1996.

14 Le texte paraît une première fois en France sous le titre suivant : La femme dans le passé, le présent et l'avenir (1879), Ravé Henri (trad.), Paris, Georges Carré, 1891. Le mariage y est critiqué comme « pire que la prostitution ». Ce texte avait heurté certains esprits en affirmant l'existence d'un instinct sexuel, y compris chez la femme.

15 Gross Otto, « La symbolique de la destruction » (1914), Etoré-Lortholary Jeanne (trad.), in Gross Otto, Psychanalyse et révolution, Le Rider Jacques (dir.), Paris, Éditions du Sandre, 2011, pp. 126-127. Ces idées sont largement partagées par Mühsam, Reventlow et les autres figures de Schwabing. Sur cette question, cf. Lucet Anatole, « Natures féminines et radicalités féministes. L'émancipation des femmes sur le Monte Verità », Cahiers d'histoire culturelle, $\mathrm{n}^{\circ}$ 29, 2018, pp. 53-66, ainsi que Lucet Anatole, Communauté et Révolution chez Gustav Landauer, Thèse de doctorat de l'université de Lyon, opérée par l'ENS de Lyon, 2018, pp. 237-277.

16 Gross Otto, « Comment surmonter la crise de la civilisation? », art. cit., p. 97. 
d'oppression existantes, Landauer défend une conception active et positive de la liberté ${ }^{17}$. Selon lui, cette dernière ne peut naître qu'au niveau des rapports entre les êtres humains, dans des alliances d'un genre nouveau, et en aucun cas au niveau du seul individu.

En outre, si Landauer assume une conception relationnelle de la liberté, il refuse de faire dépendre ces relations d'un collectif abstrait : Gross défend ainsi une liberté individuelle reposant sur une organisation communiste, tandis que Landauer prend le parti d'une liberté collective s'appuyant sur des rapports de solidarité à petite échelle, entre les individus et leurs groupements. Dans une lettre fameuse où il répond à celle qui est alors son amante et sa collaboratrice au sein de l'Alliance socialiste, Landauer revient sur la conception de l' «amour libre» dont sa correspondante s'était faite le relais :

Je veux créer de nouvelles formes de la vie partagée, parce que celles qui existent effectivement sont trop misérables, trop étroites ; ce sont les pauvres restes d'une grande époque. Mais ce serait folie de vouloir encore "supprimer» les formes du lien, les quelques-unes qui sont restées! Nous avons besoin de forme, pas d'absence de forme. Notre avons besoin de tradition, pas d'absence de cadre (Zuchtlosigkeit). ${ }^{18}$

Parmi ces formes que défend Landauer, on retrouve la figure du père que le matriarcat de Gross entendait abolir (père de trois enfants, Landauer prenait particulièrement à cœur son implication dans les tâches éducatives), la famille en tant que première forme communautaire, et au sein de celle-ci, le couple. Et si c'est bien le mariage que défend l'anarchiste contre les rapports individualistes de "l'amour libre ", on constate à la lecture de ses textes que le mariage n'a plus grand-chose à voir avec l'institution bourgeoise traditionnelle. Le mariage selon Landauer exclut ainsi le «délire possessif » du contrat d'appropriation des personnes, il bannit également toute notion de jalousie ou d'exclusivité, et ne se confond pas enfin avec une condition légale qui s'imposerait de l'extérieur aux individus ${ }^{19}$. Ce que prône Landauer en somme, c'est bien plutôt quelque chose comme un mariage, une forme d'union privilégiée entre deux êtres dont la communauté serait alors à même de se rapporter à d'autres communautés, " car pour moi la société se fonde sur les communes, et les communes sur les familles $»^{20}$. Landauer estime que cette « libre alliance »- lorsqu'elle est effectivement librement consentie - est somme toute la seule à même de servir de fondement à une société libre :

L'institution du mariage est depuis longtemps et d'une manière inextricable le fondement solide de tout ordre social pour ceci qu'elle tient bien moins au hasard et à l'artifice que les autres groupements d'appartenance commune qui s'appuient sur elle. ${ }^{21}$

17 C'est un point que ne met pas suffisamment en avant l'analyse de Paoli Guillaume, « Gustav Landauer, Erich Mühsam, Otto Gross : histoires de famille » (2014), in Gomez Freddy (dir.), Gustav Landauer, un anarchiste de l'envers, Paris, Éditions de l'éclat / À contretemps, 2018, pp. 59-86.

18 Landauer Gustav, lettre à Margarete Faas-Hardegger, $1^{\text {er }}$ avril 1909, reproduite dans Landauer Gustav, Gustav Landauer: Sein Lebensgang in Briefen, Buber Martin et Britschgi-Schimmer Ina (dir.), Frankfurt am Main, Rütten \& Loening, 1929, vol. 1/2, pp. 246-250.

19 Dans une même veine, Michel Bakounine fait de 1" « abolition non de la famille naturelle, mais de la famille légale » l'un des principes de son « Catéchisme révolutionnaire », in Bakounine Michel, Principes et organisation de la Société Internationale Révolutionnaire, Angaut Jean-Christophe (dir.), Avignon, Éditions du Chat ivre, 2013, p. 62.

20 Landauer Gustav, lettre à Paulus Klüpfel, 7 juin 1915, citée dans Wolf Siegbert, " "Nur langweilige Naturen sind frei von Widersprüchen". Gustav Landauer: Sex und Gender », in Kellermann Philippe (dir.), Anarchismus und Geschlechterverhältnisse, Lich/Hessen, Verlag Edition AV, 2016, vol. 1, § 4.

21 Landauer Gustav, « Von der Ehe », Der Sozialist, II, n 19, $1^{\text {er }}$ octobre 1910, GLAS 3.2, p. 30. 
Le double critère de l'arbitraire et de l'artifice est au cœur de l'argumentation de Landauer. Ce qui distingue selon lui les liens constitutifs de l'Alliance socialiste à laquelle il aspire, c'est une forme d'authenticité, au sens où ces liens se construiraient à partir des aspirations des différents membres, dans une perspective commune. Par opposition, les rapports contractés dans une société régie par l'amour libre - tels ceux prônés dans le cercle d'Otto Gross - se caractériseraient par leur approche consumériste, fondée sur l'intérêt de chacun plutôt que sur la construction d'un tout.

Tout en dénonçant les insuffisances et les défauts des institutions existantes, Landauer cherche donc à départager celles qui ne font que consacrer l'atomisation des êtres humains, parce que fondées sur une simple relation d'intérêt égoïste, et celles qui seraient encore porteuses de cet esprit de communauté sur lequel repose son socialisme. Loin de suivre les partisans de la table rase, Landauer estime ainsi que le changement doit se fonder sur l'existant : c'est pour cela qu'il distingue les institutions dont il s'agirait d'accélérer la chute de celles qu'il serait essentiel de conserver pour qu'advienne une révolution.

\section{Les limites du conservatisme : que retenir de Proudhon?}

Dans cette controverse avec Gross et, plus généralement, la bohème munichoise, Landauer ne part assurément pas de nulle part. Il endosse et revendique en ces matières l'héritage de celui qu'il décrivait, dans La révolution, comme «le plus grand de tous les socialistes »"22: Pierre-Joseph Proudhon. Landauer, qui fut un grand traducteur et passeur indispensable entre les mondes français et germanique, rend accessible à partir de 1909, dans les colonnes de son journal Der Sozialist, non seulement les grandes œuvres politiques du philosophe bisontin (Idée générale de la Révolution au $X I X^{e}$ siècle; Philosophie du progrès) mais encore nombre de ses articles parus dans le journal Le peuple. Pour ce qui relève plus précisément de notre propos ici, en l'occurrence le domaine des mœurs, il connaît l'ouvrage sans doute le plus sulfureux de Proudhon, La pornocratie. Il énonce en tout état de cause le concept même dans la lettre à Margarete Faas-Hardegger déjà citée plus haut, où la "pornocratie » revient à ce qui menace le socialisme, lorsque l'amour libre est pensé et pratiqué à la façon d'un Otto Gross, autrement dit selon cet individualisme de principe dont l'effet est en définitive d'affaiblir tout lien communautaire.

Il vaut la peine de soumettre à l'analyse cette relecture landauerienne de Proudhon, ne serait-ce que pour se confronter à l'évident problème de l'héritage de cette partie-là de l'œuvre du philosophe bisontin, comprenant des pages d'une faiblesse et, il faut l'affirmer sans ambages, d'une bêtise souvent consternantes. On parle donc de La pornocratie, ouvrage publié à titre posthume, mais aussi des dixième et onzième études de l'ouvrage majeur de Proudhon, De la justice dans la Révolution et dans l'Église (1858), études portant le titre " amour et mariage ». Ces textes prennent forme et sens au cœur d'une polémique initiée en 1856 dans La revue philosophique par Jenny d'Héricourt (1807-1875), auteure d'un article intitulé «M. Proudhon et la question des femmes ». La même rédigera, suite à la publication de De la justice, l'ouvrage La femme affranchie, réponse à MM. Michelet, Proudhon, E. de Girardin, A. Comte et autres novateurs modernes (1860) alors qu'une jeune polémiste, Juliette La Messine (alias Juliette Lambert, alias Mme Edmond Adam), née en 1836, avait publié en 1858 un pamphlet intitulé Idées anti-proudhoniennes sur l'amour, la femme et le mariage. La rédaction par Proudhon de La pornocratie ou les femmes dans les temps modernes constitue sa réponse définitive aux féministes l'ayant attaqué après la publication de $D e$ la justice. Si l'on devait résumer les opinions énoncées dans ce dernier ouvrage à propos de la femme, considérée par essence comme un élément passif et réceptif, et de ses attributions supposées dans la famille, restreintes à la tenue du ménage, le passage suivant tiré de la onzième étude serait éloquent, en ce qu'il sanctionne de la nécessité arithmétique ce qui ne relève en définitive que d'un pur rapport de force. Proudhon établit un rapport de 3 à 2 entre la puissance intellectuelle de l'homme et celle de la femme. Il en déduit ensuite que :

22 Landauer Gustav, La révolution (1907), Manale Margaret et Janover Louis (trad.), Arles, Sulliver, 2006, p. 116. 
puisque dans l'action économique, politique et sociale, la force du corps et celle de l'esprit concourent ensemble et se multiplient l'une par l'autre, la valeur physique et intellectuelle de l'homme sera à la valeur physique et intellectuelle de la femme, comme $3 \times 3$ est à $2 \times 2$, soit 9 à 4 . Sans doute la femme contribuant, dans la mesure qui lui est propre, à l'ordre social et à la production de la richesse, il est juste que sa voix soit entendue; seulement, tandis que, dans l'assemblée générale, le suffrage de l'homme comptera pour 9 , celui de la femme comptera pour $4:$ voilà ce que disent d'un commun accord l'arithmétique et la justice. ${ }^{23}$

L'ensemble des notations de Proudhon sur les questions dites « de mœurs » ne constituent pour l'essentiel qu'un développement de ce rapport de proportionnalité établi entre les hommes et les femmes. L'étonnant « catéchisme du mariage », au troisième chapitre de cette même onzième étude, n'intègre d'éventuelles objections que pour mieux circonscrire le rôle des femmes dans l'économie domestique: soin du ménage, éducation de l'enfance, instruction des jeunes filles sous la surveillance des magistrats, service de la charité publique. Une nouvelle fois, c'est sous le sceau de la Justice elle-même, ce principe tout autant social que moral portant tout être raisonnable à respecter la dignité en son semblable (tout en revendiquant le droit légitime d'être reconnu digne en retour) que Proudhon entérine les formes les plus pudibondes et austères de sujétion féminine. $\mathrm{Ne}$ déclare-t-il pas dans ce texte : «L'homme est travailleur, la femme ménagère : de quoi se plaindraitelle? Plus la Justice en se développant nivellera les conditions et les fortunes, plus ils se verront élevés tous les deux, celui-là par le travail, celle-ci par le ménage $»^{24}$ ? Et dans la dixième étude, revenant avec son esprit comptable sur la logique du contrat de mariage, ce sont les partisans de l'égalité des sexes qui se trouvent sommés d'avancer leurs raisons contre le mari, le chef de famille, l'homme producteur de valeur, auquel il faudrait bien plutôt tresser des louanges pour avoir eu le courage de contracter un échange inégal et «d'abdiquer sa force, en inventant, pour la femme, le mariage. $\gg\rangle^{25}$

Il est assez clair que de tels propos exigent, encore aujourd'hui, un inventaire au sein même de la tradition de pensée anarchiste. Comme nous le verrons plus loin, cette tâche est nécessaire et soulève des enjeux profonds en termes de philosophie sociale dans la mesure où il serait non seulement désinvolte mais encore strictement faux de placer la misogynie de Proudhon sur le compte de préventions idiosyncrasiques, de l'esprit du temps ou encore de la réduire à une part somme toute négligeable de son œuvre générale. La réappropriation qu'en fera Landauer montre bien plutôt en quoi les théories sur le mariage et les femmes développées par l'auteur de Qu'est-ce que la propriété ? conduisent directement au cœur de sa conception de l'ordre social. Rappelons néanmoins que dès 1857 , l'anarchiste Joseph Déjacque, exilé aux États-Unis, avait pris fait et cause pour Jenny d'Héricourt contre Proudhon, après avoir lu dans un journal américain intitulé La revue de l'Ouest un extrait de correspondance entre les deux protagonistes de la polémique qui devait se solder par la publication de La pornocratie. Dans une remarquable lettre destinée à Proudhon (et dont on a de bonnes raisons de penser qu'elle arriva à destination et fut lue) intitulée De l'êtrehumain mâle et femelle, Déjacque joue de tous les registres pour confondre le philosophe, depuis l'attaque ad hominem sur sa mentalité de vieux garçon et les effets mortifères des macérations de la chair jusqu'à des arguments politiques cruciaux sur la nécessité de tenir de front la question de l'émancipation de la femme et celle du prolétaire. Le « père Proudhon », qui parle des femmes avec une impertinence de « collégien » pour se « donner les airs de les connaître » mais qui, comme ses « adolescents auditeurs, n'en sait pas le plus petit mot», en ressort passablement éreinté, comme si son anarchisme proclamé (depuis le premier mémoire de 1840 sur la propriété) était en réalité

23 Proudhon Pierre-Joseph, De la justice dans la Révolution et dans l'Église (1860), Paris, Fayard, 1990, vol. 4/4, p. 1970.

24 Ibid., p. 2111.

25 Ibid., p. 1787. 
tronqué. En effet, dit Déjacque, si la propriété, c'est le vol, alors il faudrait avoir la probité d'appliquer ce principe pour le contrat de mariage et le rôle censément éminent tenu par l'homme dans l'économie domestique.

En d'autres termes, Déjacque exhorte Proudhon à devenir franchement « libertaire » (ce dernier terme étant exprimé publiquement pour la première fois à cette occasion) et à s'extraire de son «anarchisme juste-milieu » reproduisant à la charnière du privé et du social les systèmes de contraintes qu'il s'évertue à démanteler dans la sphère économique : « vous voulez le libre échange pour le coton et la chandelle, et vous préconisez des systèmes protecteurs de l'homme contre la femme, dans la circulation des passions humaines ; vous criez contre les hauts barons du capital, et vous voulez réédifier la haute baronie du mâle sur la vassale femelle. ${ }^{26}{ }^{26}$ Landauer connaît parfaitement cette lettre qu'il évoque en 1900 à l'occasion d'un article à propos de Déjacque, y déplorant le rapport «philistin » de Proudhon à la question des femmes ${ }^{27}$. Dans le vocabulaire de Landauer, "philistin" n'est certes pas un compliment, le terme évoquant dans une veine nietzschéenne l'individu obtus, à l'esprit pragmatique étroit, incapable d'emportements, de grandes visions et en définitive confit dans sa vie aliénée, peu importe sa classe sociale d'appartenance. Landauer, pour sa part, n'aura de cesse d'inclure toujours davantage les femmes dans le mouvement socialiste et anarchiste en exaltant les bienfaits créateurs qu'elles ne manqueraient pas d'y apporter, contrebalançant par exemple la vaine tendance masculine à la contestation pour la contestation. Il devient alors théoriquement passionnant de comprendre pourquoi, malgré tout, c'est encore de Proudhon que Landauer va s'autoriser pour exposer sa propre vision sociale et politique du mariage et de l'institution familiale, au cœur d'une défense du socialisme anarchiste.

Si l'on s'essaie à extraire le noyau théorique d'un texte comme La pornocratie, noyé par ailleurs sous les raccourcis et les insinuations les plus grossières (sur le modèle de «à femme émancipée, mari benêt »), quelques clés de compréhension s'offrent ainsi à nous. Proudhon y vitupère contre les tenants de l'égalité des sexes en leur opposant le principe fondamental (car censé reposer sur la constitution naturelle de l'homme et de la femme) de l'équivalence. Homme et femme sont différents, leur relation est en outre asymétrique (l'un parait fait pour dominer, l'autre pour recevoir passivement), mais du point de vue social ils sont équivalents car complémentaires : à l'homme la force qui s'exerce dans le domaine du travail, de la production et de la décision politique ; à la femme ce que l'auteur appelle la «beauté », principe indissolublement physique, moral et intellectuel, qui aspire la force vers l'idéal et fonde le mariage comme union de dévouement intégral. En refusant ce rapport d'équivalence et sa mise en œuvre dans l'union conjugale, les féministes qui revendiquent l'égalité desservent leur cause, en plaçant au fondement de la société un pur rapport d'intérêt, là où l'ordre républicain serait bien davantage stabilisé par des mœurs structurées autour d'une forme d'abnégation désintéressée. Aux yeux de Proudhon, c'est parce que la femme est beauté et idéal que le mariage doit être un acte d'entier dévouement. Tout calcul d'intérêt, toute recherche du plaisir sensuel ou tout amour " libre » souille au contraire l'union et la rabat sur l'adultère, le concubinage ou la prostitution : c'est l'avènement de la pornocratie, un état de déliquescence sociale. Autrement dit, on ne peut pas critiquer de façon cohérente l'esprit spéculateur, la domination des intérêts des gros capitalistes ou le bellicisme étatique si l'on tolère encore l'égoïsme et le calcul au niveau du premier maillon de l'ordre social. Voilà qui serait entièrement contraire à la justice, qui recommande de transcender perpétuellement l'intérêt bien compris. Le maintien de la justice a donc pour condition sine qua non la famille monogame, unie par le mariage de deux êtres complémentaires se vouant dévouement pour dévouement. Point

26 Déjacque Joseph, « De l'être-humain mâle et femelle. Lettre à P.-J. Proudhon » (1857), in Déjacque Joseph, $A$ bas les chefs ! Écrits libertaires (1847-1863), Bouchet Thomas (dir.), Paris, La fabrique, 2016, p. 119.

27 Landauer Gustav, « Joseph Déjacque. Zur Auferstehung eines Verschollenen », Die Zeit. Wiener Wochenschrift für Politik, Volkswirtschaft, Wissenschaft und Kunst, XXIII, nº 298, 16 juin 1900, GLAS 6.2, p. 134. 
d'échappatoire, et point d'émancipation des femmes pour Proudhon, toujours suspecte de se renverser en prostitution : « ou le mariage [...] ou la rémunération : point de milieu $»^{28}$.

Landauer, pour sa part, va considérablement amender le modèle proudhonien, d'abord par la place qu'il souhaite accorder aux femmes dans la sphère socio-économique, ensuite par une certaine libéralité dans les formes du mariage (signalée plus haut) et enfin par une reconnaissance pleine et entière du plaisir charnel comme élément non seulement engageant mais constant dans toute relation conjugale. Il n'en demeure pas moins qu'une fois évidé de ses scories les plus rétrogrades, porté par conséquent à un certain niveau d'abstraction, c'est bel et bien le modèle proudhonien de l'articulation entre le privé et le social qui est repris par Landauer. Dans sa perspective de construction du socialisme par emboîtement de niveaux, du proche au lointain, Landauer pose que l'on ne saurait faire émerger un esprit communautaire dans la société en général si ses cellules de base n'en sont pas elles-mêmes pénétrées. Ainsi compris et replacé en contexte face aux proclamations fracassantes d'un Otto Gross, la rétention de ce moment conservateur travaillé à partir de la pensée proudhonienne ne manque pas de logique. Si l'on suppose en effet des individus appelés dans la sphère privée à libérer leurs pulsions sans entraves, comme si tout cadrage équivalait à une intolérable répression, on peut légitimement se demander comment ces mêmes individus pourraient construire et incarner ensuite, dans la sphère socio-économique, quelque chose qui ressemble à du socialisme. Leur manque d'esprit commun les poussera d'autant plus logiquement à coexister tant bien que mal sous la force mécanique de l'État. C'est la raison pour laquelle Landauer en viendra dans l'Appel au socialisme à souhaiter que le foyer familial devienne le point matriciel de l'esprit d'une nouvelle culture, sans pourtant que cette dernière n'en découle directement et sans autre précision :

L'esprit fédérateur, l'alliance de plusieurs afin de faire œuvre commune, pour des raisons communes, trouve dans la famille une forme trop étroite et insuffisante pour la vie partagée. Dans la famille, il ne s'agit que d'intérêts privés. Nous avons besoin d'un noyau naturel de l'esprit commun pour la vie publique, afin que la vie publique ne soit plus comme c'était exclusivement le cas auparavant - assumée et conduite par l'État et la froideur, mais par une chaleur qui s'apparente à l'amour familial. ${ }^{29}$

Par conséquent, des êtres humains régénérés devraient être en mesure d'articuler sur un plan plus large les dispositions aux autres et aux tâches communes devenues habituelles dans la famille. Les liens vécus dans la grande communauté s'apparenteraient dès lors à un analogon de ceux qui ont cours dans la communauté familiale.

\section{La subversion subvertie : vicissitudes de la critique de la famille}

Si la position de Landauer sur la question de la famille paraissait excentrique par rapport à l'anarchisme de son époque, précisément en raison du moment conservateur dans sa réflexion, il reste intéressant de se demander ce qu'il en serait plus d'un siècle après. Nul doute qu'au début du $\mathrm{XX}^{\mathrm{e}}$ siècle dans l'Allemagne wilhelmienne, dans le cadre d'un modèle familial germanique structuré autour d'une forte prépondérance patriarcale, les thèses d'Otto Gross sur la libération de l'eros constituaient une percée incontestable en direction de l'anarchie ${ }^{30}$. Mais à la suite de deux guerres mondiales, du développement du consumérisme comme style de vie à part entière, de l'extension du spectacle au sens debordien d'une accumulation du capital à un point tel qu'il devient image et d'un culte de l'innovation technique prompt à discréditer toujours davantage

28 Proudhon Pierre-Joseph, La pornocratie ou Les femmes dans les temps modernes, Paris, Lacroix, 1875, p. 77.

29 Landauer Gustav, Aufruf zum Sozialismus, op. cit., GLAS 11, p. 139.

30 C'est un point que souligne justement Guillaume Paoli dans « Gustav Landauer, Erich Mühsam, Otto Gross : histoires de famille », art. cit. 
l'expérience des générations passées, il n'est pas vain de se demander ce qui, sous nos latitudes du moins, demeure de pertinent dans les critiques dites «freudo-marxistes » de la famille. Dès lors, l'ironie de l'histoire pourrait bien ramener sur le devant de la scène le potentiel révolutionnaire de l'anarchisme singulier de Landauer.

On doit à l'historien et critique social américain Christopher Lasch (1932-1994) d'avoir insisté dans un livre fondateur mais relativement méconnu, Haven in a Heartless World ${ }^{31}$ (1977) sur la dialectique historique des critiques de la famille, en partant précisément de remises en question du patriarcat telles que l'on pouvait les trouver chez Gross et telles qu'elles seraient développées, à partir de l'expérience nazie, par Wilhelm Reich. Rappelons que ce dernier, dans sa Psychologie de masse du fascisme, soutenait dans une veine typiquement héritée de Gross que "l'homme élevé et maintenu dans l'autoritarisme ignore les lois naturelles de l'auto-régulation, il n'a pas confiance en lui-même ; il a peur de sa sexualité parce qu'il n'a jamais appris à vivre naturellement. Il décline toute responsabilité pour ses actes et ses décisions et exige d'être tenu en laisse. ${ }^{32}$

Lasch soutient quant à lui que les discours exhortant à la libération face à l'autorité patriarcale, tenue pour le foyer de la personnalité soumise et fascisante sont petit à petit devenus, à l'encontre de leur intention initiale, les fers de lance d'une dépossession des prérogatives de la famille. À partir de l'exemple américain, qui définit toujours plus ou moins ce qui est destiné à atteindre la vieille Europe à vingt ou trente ans d'écart, il montre comment une armée d'experts et d'agents thérapeutiques se sont évertués, aussi bien matériellement que symboliquement, à déstabiliser l'expérience héritée des parents pour lui substituer un ensemble de procédures normées vouées à tenir la famille à l'abri de tout conflit délétère. Or, cette volonté de poursuivre la critique de l'autorité patriarcale dans un sens essentiellement thérapeutique a convergé historiquement, à partir des années 1920, avec le développement du capitalisme comme système culturel exhortant à la consommation comme mode de vie. Délivrée de la domination séculaire du père, la famille contemporaine se trouvait d'autant plus livrée aux forces impersonnelles du marché et à la « main visible » des agences spécialisées et des services d'État. Il en est résulté une exposition précoce des enfants à la communauté des pairs et à la société en général, ainsi qu'une émancipation dévoyée de la femme, passant bien souvent du joug de la tradition à la soumission à la tyrannie de la mode. L'ensemble offrant une singulière expression aux visions utopiques sur la communisation de la reproduction et le passage au droit matriarcal.

Dans cette approche dialectique développée dans son ouvrage de 1977, Lasch ne prétendait néanmoins pas à une absolue originalité. En réalité, il prolongeait et densifiait des pistes ouvertes par le marxisme hétérodoxe de la Théorie critique, en l'occurrence par Max Horkheimer dans son étude de 1936 intitulée Autorité et famille. Dans cet essai, si l'analyse de l'autoritarisme patriarcal dans la famille bourgeoise rappelle à bien des égards les thèses d'un Reich à la même époque, Horkheimer cherchant à comprendre les racines de la structuration psychique de l'individu autoritaire (tour à tour sadique et susceptible de se laisser aller à la soumission intégrale face à la puissance), le fondateur de l'Institut de recherches sociales préserve dans quelques passages une autre vision de la famille, conçue comme foyer d'amour. Ainsi de l'idée selon laquelle « dans le cadre de la famille, où, contrairement à la vie publique, les rapports ne sont pas dictés par le marché et où les individus ne sont pas en concurrence, l'homme a toujours eu la possibilité de n'être pas seulement une fonction, mais aussi un être humain $»^{33}$. Une telle position, que n'aurait pas reniée Landauer, était elle-même appelée à revenir dans l'œuvre de Horkheimer sous l'aspect d'une réminiscence de l'amour romantique telle qu'exprimée dans ce fragment des Notes critiques des

31 Ouvrage traduit seulement en 2012 en France, par Frédéric Joly, sous le titre Un refuge dans ce monde impitoyable. La famille assiégée, Paris, François Bourin.

32 Reich Wilhelm, La psychologie de masse du fascisme (1933), Kamnitzer Pierre (trad.), Paris, Payot, 1972 , p. 108.

33 Horkheimer Max, «Autorité et famille» in Théorie traditionnelle et théorie critique, Maillard Claude et Muller Sibylle (trad.), Paris, Gallimard, 1974, pp. 304-305. 
années 1953-1955, qu'aurait sans doute pu rédiger le philosophe anarchiste : " "L'égalité des droits de l'homme et de la femme dans le mariage", voilà qui arrange parfaitement ces messieurs d'aujourd'hui. Elle signifie que la concurrence, les human relations, bref l'aliénation, même dans ce qu'on appelle la cellule de la société, isolent encore les êtres humains les uns des autres ; qu'il ne peut plus y avoir d'utopie microscopique réalisée, de communauté jurée, pas même à la plus petite échelle, et que l'être humain est nu face à la machinerie de l'ensemble. ${ }^{34}{ }^{34}$ On constate donc que le fil, certes ténu, d'une conception subtile de la famille en ses vicissitudes historiques a donc couru pendant tout le $\mathrm{XX}^{\mathrm{e}}$ siècle, de l'anarchisme sans dogmes de Landauer au radicalisme de Lasch en passant par le marxisme hétérodoxe de Horkheimer.

La tentative de Lasch se distingue toutefois par sa capacité à saisir comment l'érosion de la famille a fini par sécréter le genre de personnalité psychique totalement adaptée à l'univers de l'illimitation capitaliste : ce qu'il appelle la personnalité narcissique, étudiée dans deux ouvrages majeurs de critique sociale, La culture du narcissisme (1979) et Le moi assiégé (1984). Ce faisant, Lasch a croisé la critique de l'économie politique et la psychanalyse en assumant, à partir de Freud et contre les interprétations d'un Otto Gross, que la conflictualité est une donnée interne à la psyché qui ne peut manquer de se déployer lorsque l'enfant grandit au contact de figures d'autorité (celles que l'on craint et qui punissent, mais aussi celles que l'on admire, qui protègent et initient). Il a précisément cherché à montrer qu'en déplaçant la tension psychique interne propre à toute éducation véritable sous la forme d'un conflit ouvert entre libido et moralité bourgeoise répressive, l'attaque contre la seconde étant censée permettre l'efflorescence de la première, les penseurs « immoralistes» tels que Gross ont prescrit le cadre théorique ôtant les derniers freins à la prolifération d'un type individuel faible, vide et en quête perpétuelle de réassurance.

En effet, livré à ses pulsions de gratification sans réelle direction pour les orienter, l'enfant grandissant dans le cadre d'une famille ainsi délabrée dans ses assises psychiques finit par retourner ses pulsions contre lui-même sous des formes agressives, réactivant les figures de domination les plus archaïques. Pour le dire autrement, l'abdication de l'autorité parentale n'entraîne pas tant un déclin du sur-moi qu'une altération de son contenu, peuplant l'inconscient du futur adulte de fantasmes agressifs et vengeurs laissant l'individu encore plus désemparé face aux manifestations réelles de l'autorité, qu'il n'aura pas appris à combattre et accepter dans le conflit familial. La personnalité dite narcissique qui en résulte se trouve alors comme un poisson dans l'eau dans l'univers mouvant de la marchandise, un pseudo-monde maternant où la distinction entre le moi et le non-moi se trouve constamment brouillée. Une société communiant dans le fétichisme de la marchandise offre le contexte pour un déploiement non entravé des pulsions de gratification, où tout se consomme et se jette à un rythme "disruptif ». Ainsi, montre Lasch, déclin de la famille et extension du capitalisme s'alimentent l'un l'autre : des êtres faibles et dépendants se lovent dans le cocon fantasmagorique du capitalisme et de ses infrastructures technologiques (mondes virtuels et réseaux sociaux en tête), lesquelles sapent davantage les possibilités d'une véritable transmission générationnelle, réduisant les familles contemporaines à des viviers de personnalités narcissiques.

À la lumière de telles réflexions, on mesure une énième fois l'étonnante plasticité du système capitaliste, sa faculté à convertir des motifs libertaires en stimulants pour ses futures mutations. À ce titre, pour les questions qui nous ont occupés au cours de cet article, on pressent qu'il n'y aurait plus guère de sens subversif à se réclamer encore aujourd'hui d'une critique de la famille patriarcale à la manière de Gross ou du freudo-marxisme qui se plaça dans son sillage. S'agirait-il pour autant de se retrancher dans une défense de la famille (sans autre précision) comme une «valeur en soi »? Landauer permet précisément de dépasser cette alternative stérile en proposant de repenser une transformation sociale à partir de ce que l'on pourrait appeler une " forme-famille », une première strate de communauté relativement ouverte quant à ses modes de relations internes et aux formes d'autorité qui y présideraient, mais suffisamment solide pour insuffler l'esprit de la communauté au

34 Horkheimer Max, Notes critiques, 1949-1969 : sur le temps présent, Cornille Sabine et Ivernel Philippe (trad.), Paris, Payot, 1993, p. 65. 
futur adulte. Rendue à ses fonctions communautaires nourricières, consistant à protéger, transmettre et éduquer à l'autonomie, la famille ne serait plus tant une «valeur » désincarnée qu'un foyer organique permettant de constituer un véritable «être communautaire (Gemeinwesen) »" , selon le mot utilisé par Martin Buber pour distinguer une communauté authentique, pénétrée par l'esprit, d'un simple agrégat d'individus de bonne volonté s'employant à faire société.

\section{Conclusion}

Si l'anarchisme est indubitablement cet « autre socialisme » encore injustement caricaturé, il est des anarchistes qui ont assumé pour eux-mêmes une excentricité par rapport à toute pétrification dogmatique de leurs idées politiques et sociales. Tel est assurément le cas de Landauer chez qui s'est sans cesse exercé le « choix révolutionnaire des éléments de l'être social qui méritent d'être conservés et qui sont valables pour une nouvelle construction $»^{36}$. Autre manière de définir un conservatisme révolutionnaire, exemplaire sur le cas de la famille, qui pourrait bien incarner le tempérament critique le plus pertinent pour notre temps. Revalorisée dans un sens landauerien, la forme-famille y aurait d'abord pour fonction de prévenir contre la formation de l'homme-masse, honni par Landauer et si précocement conformé aujourd'hui grâce à l'intrusion des dispositifs techniques au cœur de la sphère de l'intime. Ensuite, une de ses charges les plus décisives consisterait à ouvrir le nouvel arrivant dans le monde à la conscience de l'héritage et au trésor enfoui de la communauté. En ce sens, l'émergence du nouveau deviendrait solidaire de la mémoire du passé, selon un paradoxe temporel supposant que seuls des "êtres humains sains, forts et façonnés par l'esprit peuvent préparer cette nouveauté qui doit provenir de nous comme quelque chose de très ancien, si elle doit valoir quelque chose pour nous et demeurer pour nous $»^{37}$.

Ainsi constituée, en fondant sa nécessité sur ce qui la précède, son sous-sol archéologique, la cellule communautaire primaire marquerait son opposition résolue à une ère mortifère s'achevant dans le délire transhumaniste d'une abolition de la mémoire, désormais transparente et disponible comme un vulgaire étant à portée de main. Le pari philosophique de Landauer procède tout à l'inverse, en tablant sur la puissance transformatrice de l'inactuel :

Que cela soit notre socialisme : une création de l'avenir comme s'il avait été de toute éternité. Qu'il ne provienne pas des excitations et des réactions arides et virulentes du moment, mais du présent de l'esprit qui est la tradition et l'héritage de notre humanité. ${ }^{38}$

Ce qui est inactuel est difficile autant que rare. Tel est l'anarchisme de Landauer en ses moments conservateurs. Puissent ces quelques réflexions sur la famille en suggérer la pressante et si humaine nécessité.

\section{Anatole Lucet et Renaud Garcia}

35 Buber Martin, « Comment une communauté peut-elle advenir? » (1930), Cheptou Gaël (trad.), in Communauté, Paris, Éditions de l'éclat, 2018, p. 65.

36 Buber Martin, Utopie et socialisme (1950), Corset Paul et Girard François (trad.), Paris, L'Échappée, 2016 , p. 98.

37 Landauer Gustav, Aufruf zum Sozialismus, op. cit., GLAS 11, p. 105.

38 Ibid., pp. 104-105. 